\title{
Critical behavior at edge singularities in one-dimensional spin models
}

\author{
D. Dalmazi* and F. L. Sá ${ }^{\dagger}$ \\ UNESP, Campus de Guaratinguetá, DFQ, Av. Dr. Ariberto P. da Cunha, 333, CEP 12516-410 Guaratinguetá, SP, Brazil
}

(Received 25 March 2008; published 30 September 2008)

\begin{abstract}
In ferromagnetic spin models above the critical temperature $\left(T>T_{\mathrm{cr}}\right)$ the partition function zeros accumulate at complex values of the magnetic field $\left(H_{E}\right)$ with a universal behavior for the density of zeros $\rho(H)$ $\sim\left|H-H_{E}\right|^{\sigma}$. The critical exponent $\sigma$ is believed to be universal at each space dimension and it is related to the magnetic scaling exponent $y_{h}$ via $\sigma=\left(d-y_{h}\right) / y_{h}$. In two dimensions we have $y_{h}=12 / 5(\sigma=-1 / 6)$ while $y_{h}$ $=2(\sigma=-1 / 2)$ in $d=1$. For the one-dimensional Blume-Capel and Blume-Emery-Griffiths models we show here, for different temperatures, that a value $y_{h}=3(\sigma=-2 / 3)$ can emerge if we have a triple degeneracy of the transfer matrix eigenvalues.
\end{abstract}

DOI: 10.1103/PhysRevE.78.031138

PACS number(s): 05.70.Jk, 05.50.+q, 05.70.Fh

\section{INTRODUCTION}

In [1] Yang and Lee have initiated a method to study phase transitions based on partition function zeros. Since then, several applications have appeared in equilibrium and more recently in nonequilibrium statistical mechanics; see [2] for a review and references. It is expected that with the increase of computer power new applications will appear especially where analytic methods can hardly be used. In many statistical models the so-called Yang-Lee zeros (YLZs) lie on arcs on the complex $u$ plane, $u=\exp (-\beta H)$, where $H$ is an external magnetic field and $\beta=1 / K_{B} T$. The zeros at endpoints of the arcs tend to pinch the positive real axis $\operatorname{Re}(u)$ $>0$ at the critical value of the magnetic field for $T \leqslant T_{\text {cr }}$ while for $T>T_{\text {cr }}$ they accumulate at complex values $u_{E}$ $=\exp \left(-\beta H_{E}\right)$ which are called Yang-Lee edge singularities (YLESs). The linear density of zeros has a universal powerlike divergence $\rho(u) \sim\left|u-u_{E}\right|^{\sigma}$ in the neighborhood of the YLESs. The critical properties of the YLESs are described by a nonunitary $l \varphi^{3}$ field theory [3] which corresponds in two dimensions [4] to a $(p, q)=(2,1)$ nonunitary conformal minimal model leading to the prediction $\sigma=-1 / 6$. This is in agreement with numerical results for the ferromagnetic Ising model; see, e.g., $[5,6]$, and experimental magnetization data [7]. Even in the antiferromagnetic Ising model where $u_{E}$ $\in \mathbb{R}_{\text {_ }}$ numerical results [8] also lead to $\sigma=-1 / 6$. Analogously, for the ferromagnetic and antiferromagnetic $S=1 / 2$ Ising model in $d=1$ one derives exactly $\sigma=-1 / 2$. In the ferromagnetic case, the value $\sigma=-1 / 2$ has been found in other $d=1$ models such as the $n$-vector chain [9] and the $q$-state Potts model with $q>1$ and $0 \leqslant q<1$ [10]. Furthermore, in [11], by assuming that the transfer matrix eigenvalues are real in the gap of zeros and that the magnetic field is pure imaginary, one obtains $\sigma=-1 / 2$ for the $S=1,3 / 2$ Ising model and also for the Blume-Emery-Griffiths (BEG) model investigated here. The same assumptions of [11] have been made in the proof of $\sigma=-1 / 2$ given in $[12,9]$. More recently [13] another proof which holds without those assumptions has appeared in the Blume-Capel model. We show here that

\footnotetext{
*dalmazi@feg.unesp.br

†ferlopessa@yahoo.com.br
}

the latter proof contains also some hypotheses which are not satisfied in part of the parameter space of the BEG model where a critical behavior appears.

\section{GENERAL SETUP AND ANALYTIC RESULTS}

Our starting point is the couple of finite size scaling (FSS) relations, see $[14,15]$,

$$
\begin{gathered}
u_{1}(L)-u_{1}(\infty)=\frac{C_{1}}{L^{y_{h}}}+\cdots, \\
\rho(L)=C_{2} L^{y_{h}-d}+\cdots,
\end{gathered}
$$

where $C_{1}, C_{2}$ are constants, independent of the size of the lattice $L$. The Yang-Lee zero $u_{1}(L)$ is the closest zero to the edge singularity $u_{E}=u_{1}(\infty)$. The density of zeros $\rho(L)$ is calculated at $u=u_{1}(L)$. In practice we use

$$
\rho(L)=\frac{1}{N\left|u_{1}(L)-u_{2}(L)\right|},
$$

where $u_{2}(L)$ is the second closest zero to $u_{E}$ and $N$ is the total number of spins $\left(N=L^{d}\right)$. The dots in (1) and (2) stand for corrections to scaling. Our analytic method consists of deriving $y_{h}$ from an expansion for a large number of spins similar to (1) obtained directly from the transfer matrix solution. We also calculate the YLZs numerically for a finite number of spins and check the validity of (1) and (2) which furnish numerical estimates for $y_{h}$.

The partition function for the BEG model is given by [16]

$$
\begin{aligned}
Z_{N}= & \sum_{\left\{S_{i}\right\}} \exp \beta\left(J \sum_{\langle i j\rangle} S_{i} S_{j}+K \sum_{\langle i j\rangle} S_{i}^{2} S_{j}^{2}\right. \\
& \left.+\sum_{i=1}^{N}\left[H S_{i}+\Delta\left(1-S_{i}^{2}\right)\right]\right),
\end{aligned}
$$

where $S_{i}=0, \pm 1$ and the sum $\Sigma_{\langle i j\rangle}$ is over nearest-neighbor sites. We assume ferromagnetic Ising coupling $J>0$ but $K, \Delta$ can have any sign. All the couplings $J, K, \Delta$ are real while the magnetic field $H$ may be complex. The temperature is defined with respect to the ferromagnetic coupling via the compact parameter $c \equiv \exp (-\beta J)$, where $0 \leqslant T<\infty$ corre- 
sponds to $0 \leqslant c \leqslant 1$. In $d=1$, using periodic boundary conditions, $S_{i}=S_{i+N}$, the model can be easily solved via transfer matrix

$$
Z_{N}=c^{-N}\left(\lambda_{1}^{N}+\lambda_{2}^{N}+\lambda_{3}^{N}\right)
$$

where the eigenvalues $\lambda_{1}, \lambda_{2}, \lambda_{3}$ are the solutions of the cubic equation

$$
\lambda^{3}-a_{2} \lambda^{2}+a_{1} \lambda-a_{0}=0,
$$

with coefficients

$$
\begin{gathered}
a_{0}=b \tilde{x}\left(1-c^{2}\right)\left[b\left(1+c^{2}\right)-2 c\right], \\
a_{1}=b^{2}\left(1-c^{4}\right)+A \widetilde{x}(b-c), \\
a_{2}=\tilde{x}+A b .
\end{gathered}
$$

We have defined for convenience

$$
b=\exp (\beta K), \quad x=\exp (\beta \Delta), \quad \tilde{x}=x c=\exp \beta(\Delta-J),
$$

$$
A=u+1 / u=2 \cosh (\beta H) .
$$

The quantity $u=\exp (-\beta H)$ plays the role of fugacity in a lattice gas [1]. The partition function $Z_{N}$ is proportional to a polynomial of degree $2 N$ in the fugacity. Therefore, all relevant information about $Z_{N}$ is contained in its zeros $Z_{N}\left(u_{k}\right)$ $=0, k=1, \ldots, 2 N$. Due to $Z_{2}$ symmetry, $Z_{N}(u)=Z_{N}(1 / u)$, onehalf of the zeros are the inverse of the other one-half. The exact position of the YLZ can hardly be found even in one dimension. In the thermodynamic limit the partition function will vanish whenever two or more eigenvalues share the largest absolute value, see $[17,18,10]$. For a large finite number of spins the same conditions are still useful, see, e.g., [19], for locating the YLZ. Thus, following [13], let us assume that there exists a function $A(\varphi)=u(\varphi)+u^{-1}(\varphi)$ such that when we set it back in (6) we have

$$
\begin{gathered}
\lambda_{2}=e^{i \varphi} \lambda_{1}, \\
\left|\lambda_{2}\right|=\left|\lambda_{1}\right|>\left|\lambda_{3}\right| .
\end{gathered}
$$

Therefore, for a large number of spins we can neglect the contribution of $\lambda_{3}$ and write $Z_{N} \approx\left|\lambda_{1}\right|^{N}\left(1+e^{i N \varphi}\right)$. The $2 N$ YLZ are found from

$$
u^{ \pm}\left(\varphi_{k}\right)=\frac{1}{2}\left[A\left(\varphi_{k}\right) \pm \sqrt{A\left(\varphi_{k}\right)^{2}-4}\right]
$$

with $\varphi_{k}=(2 k-1) \frac{\pi}{N}, k=1,2, \ldots, N$. In order to find $A(\varphi)$ we start from the relations $a_{0}=\lambda_{1} \lambda_{2} \lambda_{3}, a_{1}=\lambda_{1} \lambda_{2}+\lambda_{1} \lambda_{2}+\lambda_{2} \lambda_{3}$, $a_{2}=\lambda_{1}+\lambda_{2}+\lambda_{3}$. Implementing the condition (10) and eliminating $\lambda_{3}$ we obtain

$$
\begin{gathered}
a_{1}=\lambda_{1} a_{2}\left(1+e^{i \varphi}\right)-\lambda_{1}^{2}\left(1+e^{i \varphi}+e^{i 2 \varphi}\right), \\
a_{0}=\lambda_{1}^{2} a_{2} e^{i \varphi}-\lambda_{1}^{3}\left(e^{i \varphi}+e^{i 2 \varphi}\right) .
\end{gathered}
$$

Manipulating (13) and (14) we further eliminate $\lambda_{1}$ and find an equation for $A(\varphi)$,

$$
\begin{gathered}
a_{0}^{2}(1+2 \cos \varphi)^{3}+4 \cos ^{2} \frac{\varphi}{2}\left(a_{1}^{3}+a_{0} a_{2}^{3}\right)-a_{1}^{2} a_{2}^{2} \\
-2(1+2 \cos \varphi)(2+\cos \varphi) a_{0} a_{1} a_{2}=0,
\end{gathered}
$$

which is the same expression obtained in [13] for the BlumeCapel model $(b=1)$. At this point, in principle, the Yang-Lee zeros are determined by (15), once we check (11). After taking the continuum limit in the corresponding solution $u\left(\varphi_{k}\right)$ one could derive the density of zeros and obtain the exponent $\sigma$. However, in practice, the expression (15) is a fourth degree polynomial equation for $A(\varphi)$ whose solutions are cumbersome in general. It is not feasible to substitute them in the solutions of the cubic equation (6), which are a bit complicated too, and then check the condition (11) for arbitrary values of the parameters $b, c, x$.

Fortunately, in order to compute the exponent $\sigma$ or $y_{h}$ we only need to study the vicinity of the YLES. The YLES is located at $A(\varphi=0)$ since at $\varphi=0$ the two, presumably, largest eigenvalues of the transfer matrix coincide $\left(\lambda_{1}=\lambda_{2}\right)$ which guarantees, see the original works $[20,21,11]$ for a recent work, the existence of a phase transition. Therefore, only the behavior of the solutions of Eq. (15) about $\varphi=0$ is required, as already noticed in [13], remembering of course that (11) must hold. Nevertheless, differently from those authors we do not take the continuum limit. By keeping $N$ finite we will be able to study the large $N$ expansion for the closest zero to the YLES and determine the exponent $y_{h}$ via comparison with (1).

In order to scan the parameter space of the BEG model for a possible critical behavior at edge singularities, we have found more useful instead of using (15) to take consecutive derivatives of expressions (13) and (14) and make some mild hypotheses as shown next.

First, from the fact that the YLES is located at $A(\varphi=0)$ it is natural to assume that the smallest phase among all $\varphi_{k}$, i.e., $\varphi_{1}=\pi / N$, corresponds to the closest zero to the YLES, namely $u_{1}(N)=u\left(\varphi_{1}\right)$. The zero $u(\pi / N)$ is determined from $A(\pi / N)$ via (12). Thus, all we need is a large $N$ expansion for $A(\pi / N)$. Our basic hypothesis is the existence of a region in the parameter space of the BEG model where we are allowed to expand $A\left(\varphi_{1}\right)$ in a Taylor ${ }^{1}$ series about $\varphi_{1}=0$,

$$
A\left(\varphi_{1}\right)=A(0)+\left.\varphi_{1} \frac{d A}{d \varphi}\right|_{\varphi=0}+\left.\frac{\varphi_{1}^{2}}{2 !} \frac{d^{2} A}{d \varphi^{2}}\right|_{\varphi=0}+\cdots .
$$

In order to proceed further we would like to collect information about $d^{n} A / d \varphi^{n}$ at $\varphi=0$. From the first derivatives of (13) and (14) we deduce

$$
\left[\tilde{x}(b-c)-b \lambda_{1}\right] \frac{d A}{d \varphi}=\left(e^{i \varphi}-1\right) \frac{\partial \lambda_{1}}{\partial \varphi}\left[\left(2+e^{l \varphi}\right) \lambda_{1}-a_{2}\right] .
$$

Assuming that $\partial \lambda_{1} / \partial \varphi$ at $\varphi=0$ is smooth enough to guarantee that the right-handed side of (17) vanishes at $\varphi=0$ we get

\footnotetext{
${ }^{1}$ Notice that due to the square root in (12), when the YLES is located at $A(0)=-2(u=-1)$, the existence of (16) does not always lead to a Taylor expansion for $u_{1}(N)$, as assumed in [13].
} 
TABLE I. Data from di-log fits of formulas (1) and (2) with $L$ $=N$ and $d=1$ for the $\Delta=-J(b=c)$ BEG model with $210 \leqslant N \leqslant 300$ spins.

\begin{tabular}{ccccc}
\hline \hline$c$ & $y_{h}^{(1)}$ & $10^{9} \chi_{(1)}^{2}$ & $y_{h}^{(2)}$ & $10^{8} \chi_{(2)}^{2}$ \\
\hline $0.1-0.5$ & 2.997 & 3.5 & 2.989 & 4.0 \\
0.6 & 2.997 & 3.5 & 2.987 & 8.3 \\
0.7 & 2.880 & $3.2 \times 10^{4}$ & 2.094 & $2.4 \times 10^{4}$ \\
$\sqrt{2} / 2$ & 1.498 & 0.9 & 1.494 & 1.3 \\
0.8 & 3.000 & 3.3 & 2.989 & 2.3 \\
0.9 & 2.997 & 3.5 & 2.989 & 3.8 \\
\hline \hline
\end{tabular}

$$
\left.\left[\tilde{x}(b-c)-b \lambda_{1}\right]_{\varphi=0} \frac{d A}{d \varphi}\right|_{\varphi=0}=0 .
$$

Therefore, if $\lambda_{1} \neq \tilde{x}(b-c) / b$ we derive $(d A / d \varphi)_{\varphi=0}=0$. Since at $\varphi=0$ we have double degeneracy $\lambda_{1}=\lambda_{2}$, back in the cubic equation (6) it is possible to show that $\lambda_{1}=\lambda_{2}=\widetilde{x}(b-c) / b$ requires $\tilde{x}=\left(1-c^{2}\right) b^{2} /|b-c|$. Thus, if $\tilde{x} \neq\left(1-c^{2}\right) b^{2} /|b-c|$ we have $^{2}(d A / d \varphi)_{\varphi=0}=0$. Consequently, the second derivatives of (13) and (14) furnish

$$
\left.\left[\widetilde{x}(b-c)-b \lambda_{1}\right]_{\varphi=0} \frac{d^{2} A}{d \varphi^{2}}\right|_{\varphi=0}=\left(\frac{\lambda_{1}}{2}\left(\lambda_{1}-\lambda_{3}\right)\right)_{\varphi=0} .
$$

Then, if there is no triple degeneracy $\lambda_{1}=\lambda_{2} \neq \lambda_{3}$ and $\tilde{x}$ $\neq\left(1-c^{2}\right) b^{2} /|b-c|$ we end up with $(d A / d \varphi)_{\varphi=0}=0$ but $\left(d^{2} A / d \varphi^{2}\right)_{\varphi=0} \neq 0$. Back in (16) and then in (12) we derive by comparison with (1) the usual result $y_{h}=2$ and $\sigma=-1 / 2$ which generalizes for the BEG model the proof of $\sigma=-1 / 2$ given in [13] for the Blume-Capel model. On the other hand, if the couplings are such that we have triple degeneracy, using $(d A / d \varphi)_{\varphi=0}=0=\left(d^{2} A / d \varphi^{2}\right)_{\varphi=0}$ it is easy to show that $\left(d^{3} A / d \varphi^{3}\right)_{\varphi=0} \neq 0$, consequently we have a critical behavior with $y_{h}=3$ and $\sigma=-2 / 3$ at the edge singularity. For completeness we mention the third case $\tilde{x}=\left(1-c^{2}\right) b^{2} /|b-c|$ which leads to $(d A / d \varphi)_{\varphi=0} \neq 0$ and $y_{h}=1$, consequently $y_{h}$ $-d=0$.

Regarding the exponent $y_{h}$, the point $u_{E}=-1[A(0)=-2]$, which corresponds to $\beta H_{E}= \pm \imath \pi$, is special as we see from (12). Due to the square root in (12) we expect a change from $y_{h}$ to $y_{h} / 2$ when the edge is located at $u_{E}=-1$. The values $y_{h}=1,2,3$ will be replaced by $y_{h}=1 / 2,1,3 / 2$, respectively. Indeed, the value $y_{h}=3 / 2$ will be confirmed numerically, see Table I (third line from the bottom) for which $\sigma=-1 / 3$ instead of $-2 / 3$, as well as $y_{h}=1$ (not presented here). The value $y_{h}=1 / 2<d$ is quite surprising since it is typical of physical phase transitions; unfortunately our numerical results based on the fit of the FSS relations (1) and (2) are less

\footnotetext{
${ }^{2}$ It is clear from the cubic equation (6) that in the special case $b$ $=c$ the condition $\lambda_{1} \neq \widetilde{x}(b-c) / b$ is always satisfied for any finite nonvanishing temperature.
}

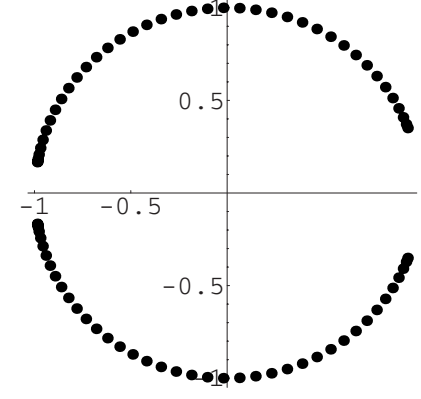

(a)

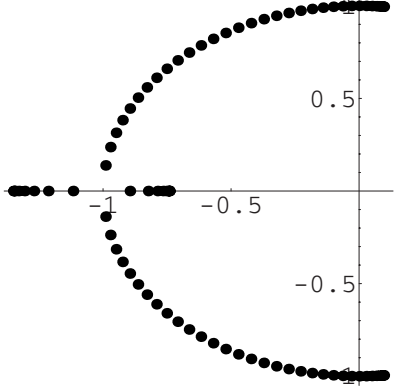

(b)
FIG. 1. Yang-Lee zeros for the BEG model with $b=c$ on the complex $u$ plane $\left(u=\exp ^{-\beta H}\right)$ at the triple degeneracy condition, see (21), and temperatures $c=0.4$ (a), $c=0.9$ (b). In both figures we have 100 zeros. The closest zero to the edge corresponds to $u_{1}^{ \pm}$ $\approx-0.98602 \pm 0.16660 i$ in (a) and $u_{1}^{+} \approx-0.74063, u_{1}^{-} \approx-1.35021$ in (b)

conclusive in this case ${ }^{3}$. It may be appropriate to mention at this point that there exists an analogous special point in the two-dimensional (2D) Ising model for which the natural variable is $\tilde{u}=u^{2}=\exp -2 \beta H$. Namely, at the point $\tilde{u}$ $=-1\left(\beta H_{E}= \pm \imath \pi / 2\right)$ there is a change, see [5], from $\sigma$ $=-1 / 6$ to $-1 / 9$ which parallels the change from $\sigma=-2 / 3$ to $-1 / 3$. Henceforth, we concentrate on the triple degeneracy case $(\sigma=-2 / 3)$.

\section{NUMERICAL RESULTS}

In this section we complement our analytic results in favor of a critical behavior with a numerical study. First of all, we note that $\lambda_{1}=\lambda_{2}=\lambda_{3}=a_{2} / 3$ holds if and only if

$$
a_{1} a_{2}-9 a_{0}^{2}=0, \quad 3 a_{1}-a_{2}^{2}=0 .
$$

For the $S=1$ Ising model $(b=1=x)$ there is no real temperature, not even in the antiferromagnetic case $(c \geqslant 1)$, at which conditions (20) hold, similarly for the $q=3$ states Potts model where $b=1 / c^{3}$ and $\tilde{x}=u / c^{3}$. However, for the BEG model there are many solutions for the triple degeneracy conditions (20). In what follows we present numerical results for two special cases.

\section{A. BEG model with $b=c(K=-J)$}

For the BEG model with $b=c(K=-J)$ the dependence of the cubic equation on the magnetic field simplifies which allows us to find the YLZ for a larger number of spins. In this case the conditions (20) imply the fine-tuning

$$
x=x(c)=\left(1+c^{2}\right) \sqrt{\left(1+c^{2}\right) /\left[27\left(1-c^{2}\right)\right]}
$$

and the position of the edge singularity, $A_{E}=\left[2\left(4 c^{2}-5\right) /(1\right.$ $\left.\left.+c^{2}\right)\right] x(c)$. The singularity is on the left-hand endpoint of an arc on the unit circle [Fig. 1(a)] for $0 \leqslant c \leqslant \sqrt{2} / 2$ and on $\mathbb{R}_{-}$

\footnotetext{
${ }^{3}$ For the cases where $y_{h}=1 / 2$, for an odd number of spins, the zeros follow a more complicated pattern (do not tend to lie on a continuous curve apparently) and the numerical uncertainties are larger.
} 
for $\sqrt{2} / 2 \leqslant c \leqslant 1$; see Figs. 1(a) and $1(\mathrm{~b})$. We have checked that the YLES on the right-hand endpoint of the arc is of the usual type $\sigma=-1 / 2$.

In finding the zeros we have started from the expression for the partition function of the BEG model obtained by a diagrammatic expansion similar to what has been done in [19] for the Blume-Capel model. Following the same steps of [19], we first define the generating function of partition functions of the BEG model on nonconnected rings and then we take its logarithm which gives rise, as is well known in diagrammatic expansions, to the generating function of only connected diagrams (usual rings). Those steps lead us to $Z_{N}=-N c^{-N}\left[\ln \left(1-a_{2} g+a_{1} g^{2}-a_{0} g^{3}\right)\right]_{g^{N}}$. The notation $[f(g)]_{g^{N}}$ indicates the power $g^{N}$ in the Taylor expansion of $f(g)$ about $g=0$. The constant $g$ plays the role of a coupling constant in a diagrammatic expansion from Feynman (perturbation theory). The above expression for $Z_{N}$ is equivalent to (5) for an arbitrary number of spins. In particular, the reader can easily check it for a low number of spins by using the relationships between eigenvalues and coefficients of the cubic equation given in the text between formulas (12) and (13). The expression turns out to be more efficient than (5) from a computational point of view. In practice, we have used rings with $N_{a}=210+10(a-1)$ spins where $1 \leqslant a \leqslant 10$. The zeros have been found with the help of MATHEMATICA software. They agree up to the first 30 digits with few cases where they are known exactly as, for instance, $\tilde{x}=c\left(3-c^{2}\right)^{2} / 2$ and $b$ $=c\left(3-c^{2}\right) / 2$ where, for an even number of spins, the YangLee zeros are exactly located at $A_{k}=-2-2 i\left(1-c^{2}\right) \sin [2 \pi(1$ $+3 k) / 3 N], k=0, \ldots, N-1$.

According to the discussion at the end of the preceding section, we expect $y_{h}=3 / 2$ at $c=\sqrt{2} / 2$ where $A_{E}=-2$, and $y_{h}=3$ elsewhere. This is approximately confirmed in Table I. The estimates $y_{h}^{(1)}$ and $y_{h}^{(2)}$ refer, respectively, to di-log fits of (1) and (2) with $L=N$ and $d=1$. We take the logarithm of the absolute value of the imaginary (real) part of (1) for $0 \leqslant c$ $\leqslant \sqrt{2} / 2(\sqrt{2} / 2<c \leqslant 1)$ and neglect corrections to scaling in (1) and (2). For both fits we have calculated $\chi^{2}$ and the deviation from the ideal $( \pm 1)$ Pearson coefficient, but since they have the same temperature dependence we only display $\chi^{2}$ in our tables. For $0.1 \leqslant c \leqslant 0.5$ the results are quite homogenous but the proximity of the point $\sqrt{2} / 2 \approx 0.707$ leads to a crossover behavior at $c=0.7$ with an increase of 4 orders of magnitude in $\chi^{2}$.

Alternatively, from (2) with $d=1$ and $L=N$, neglecting corrections, we obtain ${ }^{4}$

$$
y_{h}^{(\rho)}\left(N_{a}\right)=1+\left(\ln \frac{N_{a+1}}{N_{a}}\right)^{-1} \ln \left(\frac{\rho\left(N_{a+1}\right)}{\rho\left(N_{a}\right)}\right), \quad a=1,2, \ldots, 9 .
$$

Some results are shown in Table II altogether with the $N$ $\rightarrow \infty$ Bulirsch-Stoer (BST) [22,23] extrapolation. We remark that in the BST approach there is a free parameter $w$ which depends on how we approach the $N \rightarrow \infty$ limit, namely,

\footnotetext{
${ }^{4} \mathrm{~A}$ similar formula can be deduced from (1) but it gives results even closer to $y_{h}=3$.
}

TABLE II. Finite size results, obtained from (22), for the magnetic scaling exponent $y_{h}$ for the $(b=c)$ BEG model with $210 \leqslant N$ $\leqslant 300$ spins at temperatures $c=0.6$ and $c=0.7$.

\begin{tabular}{lcc}
\hline \hline$N_{a}$ & $y_{h}^{(\rho)}(c=0.6)$ & $y_{h}^{(\rho)}(c=0.7)$ \\
\hline 210 & 2.98450354337 & 1.96268002086 \\
220 & 2.98540244520 & 1.99853987788 \\
230 & 2.98619971860 & 2.03403890400 \\
240 & 2.98691163425 & 2.06907936446 \\
250 & 2.98755121168 & 2.10357440914 \\
260 & 2.98812897995 & 2.13744702199 \\
270 & 2.98865353760 & 2.17062929861 \\
280 & 2.98913197003 & 2.20306194085 \\
290 & 2.98957016460 & 2.23469387995 \\
$\infty$ & $3.000009(6)$ & $3(8)$ \\
\hline \hline
\end{tabular}

$y_{h}(N)=y_{h}(\infty)+\frac{A_{1}}{N^{w}}+\frac{A_{2}}{N^{2 w}}+\cdots$. The BST algorithm approximates the original function $y_{h}(N)$ by a sequence of ratios of polynomials with a faster convergence than the original sequence $y_{h}\left(N_{a}\right)$. Following [23] we can determine $w$ in such a way that the estimated uncertainty of the extrapolation is minimized. By defining the uncertainty as 2 times the difference between the two approximants just before the final extrapolated result, see [23], we have tested $w$ in the range $0.5 \leqslant w \leqslant 2.5$ and concluded that $w=1$ is the best choice. All BST results in this work have been calculated at $w=1$. For $0.1 \leqslant c \leqslant 0.6$ the extrapolations are remarkably close to $y_{h}$ $=3$. The huge uncertainty at $c=0.7$ (last row in the third column in Table II) signalizes again the crossover to $y_{h}$ $=3 / 2$. Our numerical results confirm both finite size scaling relations (1) and (2). See Fig. 2 for a plot regarding the relation (2).

Before we go to the next case we can, without much numerical effort, check that the triple degeneracy conditions lead in fact to new results. When we choose $b=c$ the equation (15) becomes a cubic equation for $A(\varphi)$. By further fixing $x=x(c)$ according to (21) and $c=0.4$, for sake of comparison with the zeros in Fig. 1, we find three solutions

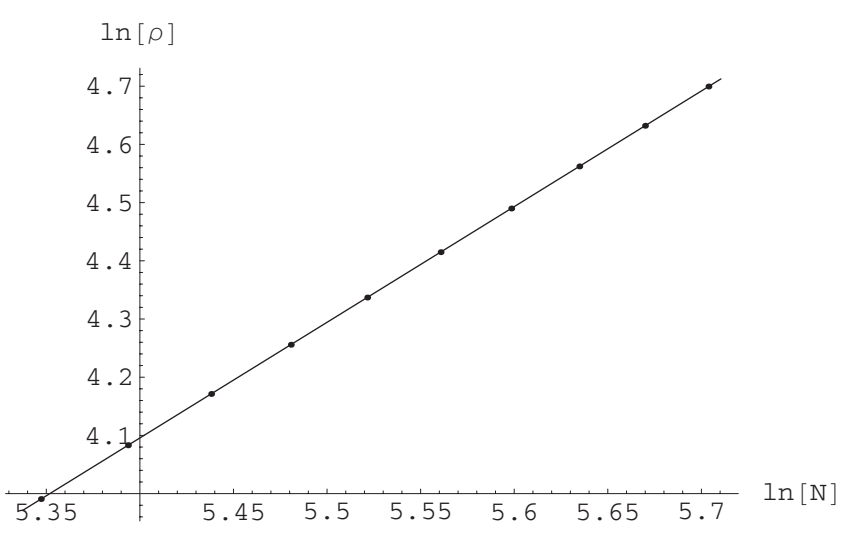

FIG. 2. Di-log fit for the density of zeros around the edge singularity for the $\Delta=-J(b=c)$ BEG model. We have used $210 \leqslant N$ $\leqslant 300$ spins at temperature $c=0.6$. The solid line is $\ln \rho$ $=1.98724(\ln N)-6.63547$. 


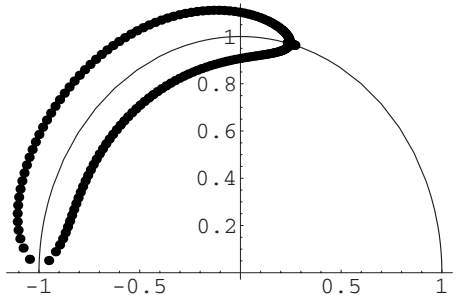

(a)

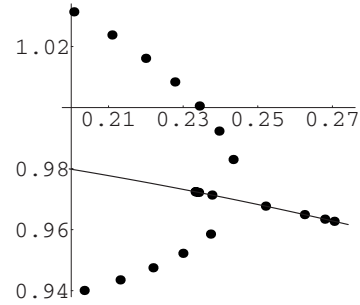

(b)

FIG. 3. One-half $[\operatorname{Im}(u)>0]$ of the Yang-Lee zeros for the Blume-Capel model $(b=1)$ on the complex $u$ plane $\left(u=\exp ^{-\beta H}\right)$ at the triple degeneracy condition, see (26), temperature $c=0.5$ and $N=150$ spins. The solid line in both figures is part of the unit circle. The closest zero to the edge corresponds to $u_{1}^{+} \approx 0.2332+0.9734 i$ as shown in detail in (b).

$A_{i}(\varphi), i=1,2,3$ which are still complicated, but expanding about $\varphi=0$ we derive

$$
A_{1}(\varphi)=\frac{829}{900} \sqrt{\frac{29}{7}}-\frac{9 \sqrt{203}}{400} \varphi^{2}+\frac{\sqrt{203}}{1440} \varphi^{4}+O\left(\varphi^{6}\right),
$$

$$
A_{2}(\varphi)=-\frac{218}{225} \sqrt{\frac{29}{7}}+\frac{1}{75} \sqrt{\frac{203}{3}} \varphi^{3}-\frac{\sqrt{203}}{450} \varphi^{4}+O\left(\varphi^{5}\right)
$$

$$
A_{3}(\varphi)=A_{2}(-\varphi)
$$

Now we see that the symmetry $\varphi \rightarrow-\varphi$ of Eq. (15) mentioned in [13] does not necessarily lead to even solutions. The operation $\varphi \rightarrow-\varphi$ exchange the second with the third solution. The first solution $A_{1}(\varphi)$ is the usual Yang-Lee edge singularity and gives $\sigma=-1 / 2$ while the second one leads to $\sigma=-2 / 3$. The second solution corresponds to the edge singularity appearing on the left-hand side of the arcs in Fig. 1(a). The third solution is not a true singularity. We can, even before the expansion about $\varphi=0$, substitute $A_{i}(\varphi)$ in the eigenvalue solutions of the cubic equation (6) and check numerically that the magnitude of two eigenvalues degenerates for all $A_{i}(\varphi)$. However, for $A_{3}(\varphi)$ such magnitude is smaller

TABLE III. Data from di-log fits of formulas (1) and (2), with $L=N$ and $d=1$, for the Blume-Capel model with $96 \leqslant N \leqslant 150$ spins and temperatures $0.3 \leqslant c \leqslant 0.9$. We have used $\left|\operatorname{Re}\left[u_{1}^{+}(N)-u_{1}(\infty)\right]\right|$ in the di-log fit of (1).

\begin{tabular}{lcccc}
\hline \hline$c$ & $y_{h}^{(1)}$ & $10^{5} \chi_{(1)}^{2}$ & $y_{h}^{(2)}$ & $10^{3} \chi_{(2)}^{2}$ \\
\hline 0.3 & 3.200 & 5.03 & 4.612 & 3.10 \\
0.4 & 3.148 & 2.52 & 3.795 & 2.10 \\
0.5 & 3.119 & 1.51 & 3.561 & 0.72 \\
0.6 & 3.100 & 1.00 & 3.442 & 0.36 \\
0.7 & 3.088 & 0.76 & 3.370 & 0.22 \\
0.8 & 3.078 & 0.58 & 3.320 & 0.15 \\
0.9 & 3.071 & 0.48 & 3.285 & 0.11 \\
\hline \hline
\end{tabular}

TABLE IV. Finite size results, obtained from (22), for the magnetic scaling exponent $y_{h}$ for the Blume-Capel model $(b=1)$ with $96 \leqslant N \leqslant 150$ spins at temperatures $c=0.3$ and $c=0.9$.

\begin{tabular}{lcc}
\hline \hline$N_{a}$ & $y_{h}^{(\rho)}(c=0.3)$ & $y_{h}^{(\rho)}(c=0.9)$ \\
\hline 96 & 6.65138835174 & 3.36602639165 \\
102 & 5.40615603620 & 3.33723696161 \\
108 & 4.85302919346 & 3.31271060187 \\
114 & 4.52543971437 & 3.29155556642 \\
120 & 4.30452579272 & 3.27311462040 \\
126 & 4.14377246532 & 3.25689223008 \\
132 & 4.02074088023 & 3.24250742353 \\
138 & 3.92311802198 & 3.22966230906 \\
144 & 3.84352213555 & 3.21812047991 \\
$\infty$ & $3.001(1)$ & $3.00000(8)$ \\
\hline \hline
\end{tabular}

than the real (nondegenerate in magnitude) eigenvalue and so it does not satisfy (11).

\section{B. Blume-Capel model $(b=1)$}

For the Blume-Capel model $K=0(b=1)$ the triple degeneracy conditions (20) lead to complicated formulas for both the fine-tuning function $\tilde{x}=\tilde{x}(c)$ and the position of the edge $A_{E}$,

$$
\begin{gathered}
\tilde{x}(c)=\left\{1-8 c+10 c^{2}+10 c^{3}-9 c^{4}+3\left(1-c^{2}\right)\right. \\
\left.\times\left[(P+Q)^{1 / 3}+R(P+Q)^{-1 / 3}\right]\right\}^{1 / 2}, \\
A_{E}=\frac{\tilde{x}(c)\left[(1+c)\left(5-15 c+5 c^{2}+3 c^{3}\right)+\tilde{x}^{2}(c)\right]}{(1+c)\left(1+c^{2}\right)+\tilde{x}^{2}(c)(2-3 c)},
\end{gathered}
$$

where $P=\sqrt{c^{3}\left(1+c-c^{2}\right)\left(1-3 c-c^{2}\right)^{2}}, \quad Q=c^{2}\left(5-36 c+95 c^{2}\right.$ $\left.-90 c^{3}+27 c^{4}\right)$, and $R=c\left(9 c^{3}-20 c^{2}+10 c-1\right)$. In the whole range of temperatures $0 \leqslant c \leqslant 1$ the function $\tilde{x}(c)$ given in from (26) runs +1 to +2 ; in fact we have approximately ${ }^{5}$ $\tilde{x}(c) \approx 1+c$. The position of the zeros $A_{E}$ ranges from -2 to +2 which guarantees, see (9), that the edge is always on the unit circle on the complex $u$ plane. However, the finite size numerical calculations reveal that the zeros on the unit circle correspond to a small fraction of a whole set of zeros, see Fig. 3. Those zeros tend to form a short arc whose right-hand endpoint is a usual YLES with $\sigma=-1 / 2$ as we have checked. The unusual behavior appears on the left-hand endpoint, whose results are displayed in Tables III and IV. There is no edge singularity for the other zeros outside the unit circle. For technical numerical reasons we have used $N_{a}=96+(a$ $-1) 6$ spins with $1 \leqslant a \leqslant 10$ and $0.3 \leqslant c \leqslant 0.9$. Although the finite size results move away from $y_{h}=3$ as $T \rightarrow 0$ (Table III),

\footnotetext{
${ }^{5}$ Although $\tilde{x}(c) \approx 1+c$ it is important that $\tilde{x}(c) \neq 1+c$ since $\tilde{x}$ $=\left(1-c^{2}\right) b^{2} /|b-c|=1+c$ for $b=1$ and, as we have discussed after formula (18), at that point we may no longer have $d A / d \varphi=0$ at $\varphi$ $=0$. In fact, we have confirmed numerically that $y_{h} \approx 1$ at this point. It corresponds to a bifurcation point where one curve becomes two disjoint arcs of zeros; see Fig. 2(c) of [19].
} 
the BST extrapolations (Table IV) all tend to $y_{h}=3$ with increasing uncertainty as $T \rightarrow 0$. In Table IV we only display the limit cases $c=0.3$ and $c=0.9$. Since we have noticed that the fraction of zeros on $S_{1}$ decreases as $T \rightarrow 0$, our interpretation is that we are closer to a continuum distribution of zeros as the temperature increases and the temperature dependence of $y_{h}^{(\rho)}(N)$ is a pure finite size artifact. In conclusion, our numerical results are in good agreement with our analytic predictions.

Analogously to what we have done at the end of the preceding section, if we now assume $b=1, \tilde{x}=\tilde{x}(c)$ as given in (26) and fix some specific value for the temperature, we show that the expansions of the solutions of the equation (15) around $\varphi=0$ give both $\sigma=-1 / 2$ and $\sigma=-2 / 3$.

\section{CONCLUSION}

We have derived for the Blume-Capel and BEG models, analytically and numerically, a value for the magnetic scaling exponent $y_{h}=3$ and edge exponent $\sigma=-2 / 3$. This is only possible due to a triple degeneracy of the transfer matrix eigenvalues. We have checked that our di-log fits, see, e.g., Fig. 2, are consistent with the finite size scaling relations (1) and (2) which appear around the usual Yang-Lee edge singularity. We stress that the critical behavior found here is not in conflict with previous numerical and analytic results in the literature. In particular, the proof of $\sigma=-1 / 2$ given in $[12,9,11]$ assumes that in the gap of zeros close to the YLES all eigenvalues are real. In the examples that we have analyzed so far we have verified that this is indeed the case for the usual YLES $(\sigma=-1 / 2)$; however, only the largest eigenvalue is real in the gap close to the singularity $(\sigma=-2 / 3)$.
Besides, although all eigenvalues are real at vanishing magnetic field, as one can check from (6) at $A=2$, apparently nothing prevents the two eigenvalues with smallest magnitude of acquiring an imaginary part before reaching the edge singularity as we increase the magnetic field through imaginary values. Consequently, so far we have not been able to prove that the edge singularity closer to $\mathbb{R}_{+}$is always of the usual type $(\sigma=-1 / 2)$, or alternatively, that the triple degeneracy condition could be satisfied also by an edge singularity close to the positive real axis. Concerning the recent proof of $\sigma=-1 / 2$ given in [13], it supposes that $\left(d^{2} u / d \varphi^{2}\right)_{\varphi=0} \neq 0$ which is not the case in the examples presented here where $\lambda_{1}=\lambda_{2}=\lambda_{3} \neq \tilde{x}(b-c) / b$.

Finally, it is important to remark that instead of imposing the triple degeneracy conditions (20) and finding rather peculiar fine-tuning functions (21) and (26) we could turn the argument around and claim that for some given values of the couplings $b$ and $x$ we can, in many cases, find a specific real temperature $c$ such that conditions (20) are satisfied and the usual result $\sigma=-1 / 2$ is replaced by $\sigma=-2 / 3$. Since the number of transfer matrix eigenvalues is infinite for $d>1$, even for the $S=1 / 2$ Ising model, we might speculate that the degeneracy of more than two largest eigenvalues could play a role also in higher dimensional spin models and lead to a change in the exponent $\sigma$ at specific temperatures in $d>1$.

\section{ACKNOWLEDGMENTS}

D.D. is partially supported by CNPq and F.L.S. has been supported by CAPES. D.D. has benefited from discussions with A. S. Castro, R. V. de Moraes, A. de Souza Dutra, and M. Hott. A discussion with N. A. Alves on the BST extrapolation method is gratefully acknowledged. Special thanks go to Luca B. Dalmazi for some computer runs.
[1] C. N. Yang and T. D. Lee, Phys. Rev. 87, 404 (1952); T. D. Lee and C. N. Yang, ibid. 87, 410 (1952).

[2] I. Bena, M. Droz, and A. Lipowski, Int. J. Mod. Phys. B 19, 4269 (2005).

[3] M. E. Fisher, Phys. Rev. Lett. 40, 1610 (1978).

[4] J. L. Cardy, Phys. Rev. Lett. 54, 1354 (1985).

[5] V. Matveev and R. Shrock, Phys. Rev. E 53, 254 (1996).

[6] S.-Y. Kim, Phys. Rev. E 74, 011119 (2006).

[7] C. Binek, Ising-type Antiferromagnets (Springer-Verlag, Berlin, 2003); Phys. Rev. Lett. 81, 5644 (1998).

[8] S.-Y. Kim, Nucl. Phys. B 705, 504 (2005).

[9] D. A. Kurze, J. Stat. Phys. 30, 15 (1983).

[10] Z. Glumac and K. Uzelac, J. Phys. A 27, 7709 (1994).

[11] X.-Z. Wang and J. S. Kim, Phys. Rev. E 58, 4174 (1998).

[12] M. Fisher, Suppl. Prog. Theor. Phys. 69, 14 (1980).
[13] R. G. Ghulghazaryan, K. G. Sargsyan, and N. S. Ananikian, Phys. Rev. E 76, 021104 (2007).

[14] C. Itzykson, R. B. Pearson, and J. B. Zuber, Nucl. Phys. B 220, 415 (1983).

[15] R. J. Creswick and S.-Y. Kim, Phys. Rev. E 56, 2418 (1997).

[16] M. Blume, V. J. Emery, and R. B. Griffiths, Phys. Rev. A 4, 1071 (1971).

[17] T. S. Nielsen and P. C. Hemmer, J. Chem. Phys. 46, 2640 (1967).

[18] S. Katsura and M. Ohminami, J. Phys. A 5, 95 (1972).

[19] L. A. F. Almeida and D. Dalmazi, J. Phys. A 38, 6863 (2005).

[20] E. N. Lassettre and J. P. Howe, J. Chem. Phys. 9, 747 (1941).

[21] J. Ashkin and W. E. Lamb, Phys. Rev. 64, 159 (1943).

[22] R. Bulirsch and J. Stoer, Numer. Math. 6, 413 (1964).

[23] M. Henkel and G. Schutz, J. Phys. A 21, 2617 (1988). 